\title{
Cisj
}

\section{FACE DETECTION ON GRAYSCALE AND COLOR IMAGES USING COMBINED CASCADE OF CLASSIFIERS}

\author{
Yuriy Kurylyak ${ }^{1)}$, Ihor Paliy ${ }^{1)}$, Anatoly Sachenko ${ }^{1)}$, Amine Chohra ${ }^{2)}$, Kurosh Madani ${ }^{2)}$ \\ 1) Research Institute of Intelligent Computer Systems, \\ Ternopil National Economic University, \\ 3 Peremoga Square, 46004, Ternopil, Ukraine \\ \{yuk,ipl, as\}@tneu.edu.ua \\ 2) Images, Signals and Intelligent Systems Laboratory (LISSI / EA 3956), \\ PARIS XII University, Senart-FB Institute of Technology \\ Av. Pierre Point, Bat. A, F-77127, Lieusaint, France \\ \{chohra,madani\}@univ-paris 12.fr
}

\begin{abstract}
The paper describes improved face detection methods for grayscale and color images using the combined cascade of classifiers and skin color segmentation. The combined cascade with proposed face candidates'verification method allows achieving one of the best detection rates on CMU test set and a high processing speed suitable for a video flow processing. It's also shown that the mixture of color spaces is more efficient during the skin color segmentation than the application of one color space. A lot of experiments are made to choose rational parameters for the developed face detection system in order to improve the detection rate, false positives' number and system's speed.
\end{abstract}

Keywords: Face Detection, Skin Color Segmentation, Haar-like Features’ Cascade of Weak Classifiers, Convolutional Neural Network, Combined Cascade of Classifiers.

\section{INTRODUCTION}

During the last decade the researches in the face detection (FD) area became more and more active. The reason is that there are a lot of applications of this task such as face recognition, videoconferences, content-based image retrieval, video surveillance, human-computer interface, face expressions' analysis, visitors' counting, access control where the detection is the first stage of any face processing [1]. The FD task is quite easy for the humans but becomes a serious problem while developing an automatic detection system. In this case it is necessary to deal with a lot of factors which affect the face appearance such as viewpoint, slope, face expression, occlusions, light conditions, etc.

There are number of existing FD approaches but in respect to the detection rate and false positives' number (FP) more effective are the methods from appearance-based group [1]. Some of them like neural networks $[2,3,4,5]$, support vector machines $[6,7,8]$, Bayesian classifier [9] make use of the monolithic classifiers and some of them are based on the cascade of classifiers [10,11, 12, 13, 14, 15].

In general, the FD methods based on the face modeling show good results on the complex test sets (for example, on CMU test set [3] which includes 130 images with 507 faces) and are able to process more than 10 images per second. There is also the range of applications where the existent FD methods are not suitable, for example, video surveillance systems and access control. In such systems to achieve the high detection rate with the lowest false positive detections it is necessary to use the complex monolithic classifiers. However, their usage with the existing face search strategies lowers the performance of FD subsystem and makes impossible processing of the video stream. Methods with the cascade of classifiers allow fast video information processing but are not so effective. For example, with increasing of the detection rate by more than $90 \%$ the false positive detection rate increases exponentially [12]. Thus, the development of new FD methods that will show a high face detection rate and low number of false positive detections is very actual task. The important point also is the possibility to work with the video stream.

In this paper we research the proposed in $[16,17]$ FD methods for effective processing of grayscale and color images by applying the skin color segmentation and combined cascade of classifiers. 
Moreover, in order to configure the rational parameters of developed FD system we provide the experimental results of such researches in respect to the detection rate, number of false positive detections as well as the performance.

\section{FACE DETECTION ON GRAYSCALE IMAGES}

The cascade structure of the FD classifier allows achieving high image processing speed due to the fast background rejection and paying more attention to the face-like regions. But in comparison with the monolithic classifiers the cascade classifier (for example, Haar-like features' cascade of weak classifiers [10]) increases the detection error and FP rate. The extension of Haar-like features' set [12] as well as improvement of the training algorithm [11] for the cascade of weak classifiers allows increasing the detection rate only by $7-8 \%$ for the low FP rate. Therefore, to achieve higher detection and lower FP rates it is necessary to join the quick cascade classifier and accurate monolithic one within the two-level combined cascade of classifiers instead of using them independently. The two-level cascade of classifiers is called "combined" because it combines the different-nature classifiers, which are chosen and justified by authors: the first level is represented by the Haar-like features' cascade of weak classifiers, which is responsible for the face candidates' detection, and the second level is a convolutional neural network for the candidates' verification (Fig. $1)$. We should also mention that there is no input image preprocessing stage as well as in [5].

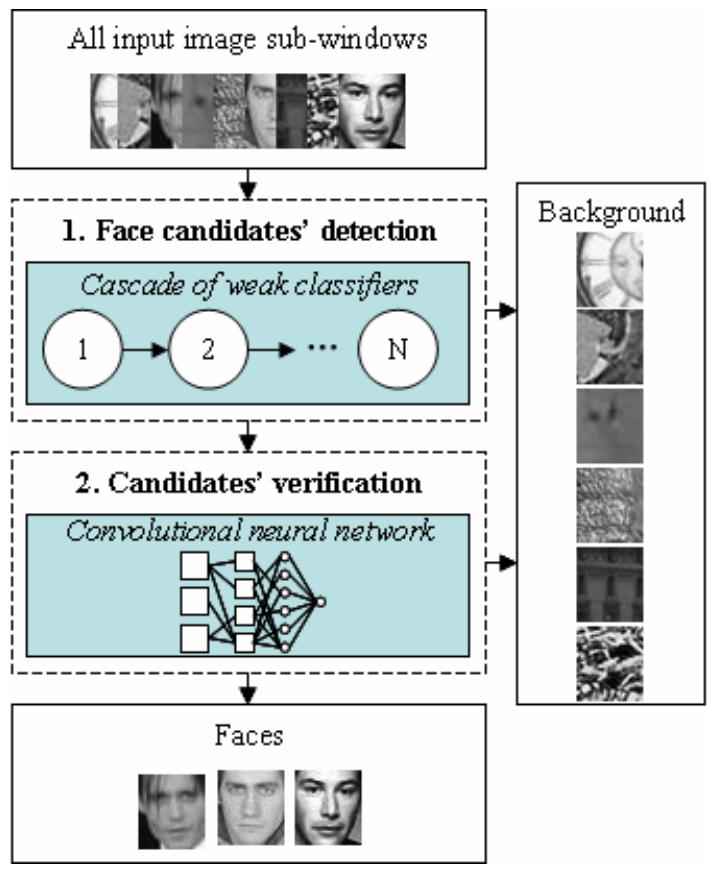

Fig. 1 - Face detection process using combined cascade of classifiers.
The Haar-like features' cascade of weak classifiers (CWC) [10] allows detecting face candidates very quickly (near real-time mode for the video flow). The CWC consists of levels which include one or more weak classifiers. The weak classifier's input is represented by Haar-like feature of the rectangular form which is composed of "white" and "black" rectangles. The feature's value is [10]

$$
f(x)=w_{w} \times S_{w}+w_{b} \times S_{b},
$$

where $x$ - input image sub-window, $w_{w}$ and $w_{b}-$ whole rectangle's and its black part's weights accordingly, $S_{w}$ and $S_{b}$ - whole rectangle's and its black part's sums of pixels (these sums are calculated very fast using an integral image [10]). A weak classifier's output value defines as

$$
h(x)=\left\{\begin{array}{c}
1, \text { if } p \times f(x)<p \times \theta \\
-1, \text { otherwise }
\end{array},\right.
$$

where $p$-polarity, which indicates the direction of the inequality, $\theta$ - weak classifier's threshold. The cascade of weak classifiers is a linear combination of weak classifiers which is evaluated as [10]

$$
H(x)=\sum_{t=1}^{T} w_{t} \times h_{t}(x),
$$

where $T$ - weak classifiers' number, $w_{t}-t$-weak classifier's weight. The AdaBoost algorithm [18] is used for the CWC's training and the selection of the most important Haar-like features.

The verification level uses the convolutional neural network $(\mathrm{CNN})$ [5] which is more robust to the input images' deformations (shifts, scales, rotations, occlusions) in the classification issues due to the architectural features than other known classifiers [19]. The output value of a neuron with the coordinates $(m, n)$ of $p$-plane and l-layer is [16]

$$
y_{m, n}^{l, p}(x)=F\left(S_{m, n}^{l, p}(x)\right),
$$

where $x$ - input face candidate's image, $F$ - neuron's transfer function, $S_{m, n}^{l, p}(x)$ - neuron's weighted sum. According to the recommendation in [20] the bipolar sigmoid transfer function is used for CNN with the output range $[-1 ; 1]$. Therefore, the expression (1) may be represented as

$$
y_{m, n}^{l, p}(x)=\frac{2}{1+\exp \left(-S_{m, n}^{l, p}(x)\right)}-1,
$$

where a neuron's weighted sum is: 


$$
S_{m, n}^{l, p}(x)=\left(\sum_{k=0}^{K-1} \sum_{r=0}^{R-1} \sum_{c=0}^{C-1} y_{2 m+r, 2 n+c}^{l-1, k}(x) \times w_{r, c}^{l, p, k}\right)-b^{l, p},
$$

where $K$ - input planes' number (as well as convolutional kernels), $R$ and $C$ - convolutional kernel's height and width, $w_{r, c}^{l, p, k}-$ synaptic weight with coordinates $(r, c)$ in the convolutional kernel between $k$-plane of the (l-1)-layer and $p$-plane of the $l$-layer, $b^{l, p}-$ neurons' threshold of the $p$-plane and l-layer.

We used the sparse structure of the CNN instead of the full-connected as well as decreased the number of layers by performing the convolution and subsampling operations in each plane simultaneously [21] (Fig. 2) in order to increase the neural network's processing speed.

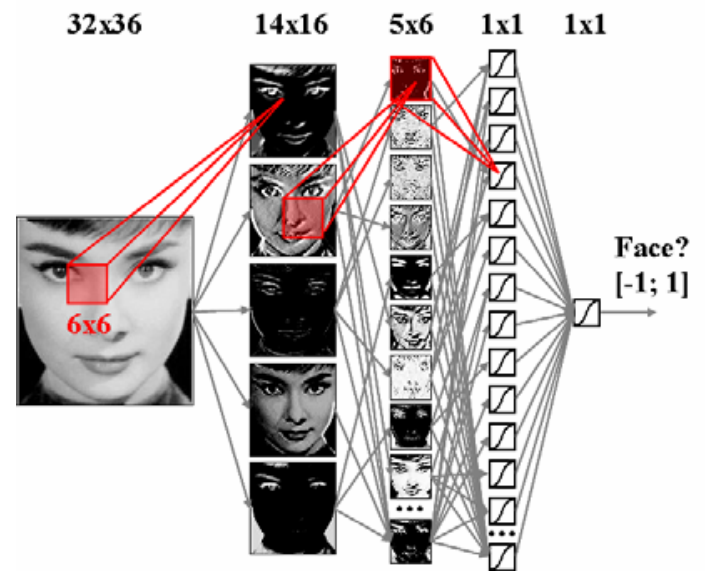

Fig. 2 - Convolutional neural network's structure for the combined cascade of classifiers.

In order to define the way of interaction between the levels of the combined cascade of classifiers we analyzed and compared two known face candidates' verification methods [5, 10-15]:

- straight verification of candidate's image by CNN,

- extended candidate's image is scanned by CNN using fixed-size window at several scale levels.

The face candidate's image is a clustering result of all multiple detections across scale and position for one candidate.

The first known verification method is simple in implementation and very fast because the number of CNN's simulations is equal to the quantity of candidates. It's used in some FD approaches with cascades of single-type classifiers: weak classifiers based on Haar-like features [10], multilayer feedforward neural networks with local receptive fields [14], SNoW networks [15] etc. The authors' researches showed [16] that this verification method applied to the combined cascade of classifiers results in the lower detection rate $(70.5 \%$ with $10 \mathrm{FP}$ on CMU test set) in comparison with the FD method of P. Viola and M. Jones [10]. The detection rate increases $(83.5 \%$ with $10 \mathrm{FP})$ [16] when the first known method is applied with the verification of all candidate's multiple detections. But the verification time also increases because the number of CNN's simulations for one face candidate defines as:

$$
\operatorname{NNSim}(x)=\sum_{d=0}^{D-1} W I^{d}(x),
$$

where $\operatorname{WIN}^{d}(x)-d$-multiple detection across scale or position for the face candidate $x, D$ - the number of multiple detections.

The second known verification method allows to accept face candidates even in the case of inaccuracy detection of their coordinates across scale or position $[3,5]$ and this fact is acknowledged by a high detection rate of the combined cascade of classifiers ( $88.8 \%$ with 9 FP on CMU test set). But authors also ascertained that the mentioned verification method is characterized by the higher computational complexity than the first known method because the number of CNN's simulations for each face candidate is

$$
\operatorname{NNSim}(x)=\sum_{s=0}^{S-1} \sum_{r=0}^{R-W} \sum_{c=0}^{C-H} W I N_{r, c}^{s}(x),
$$

where $\operatorname{WIN}_{r, c}^{s}(x)$ - a fixed-size window with the coordinates $(r, c)$ on the face candidate's image $x$ for $s$-scale level, $W$ and $H$ - window's width and height, $R$ and $C$ - width and height of a face candidate's image.

Thus two above mentioned known face candidates' verification methods have some disadvantages which don't allow their efficient applying to the combined cascade of classifiers: the first method has a low detection rate and the second one is characterized by a high computational complexity. Therefore, the authors proposed a new verification method (Fig. 3). It is based on the CNN's property to process an input image of any size at once [5].

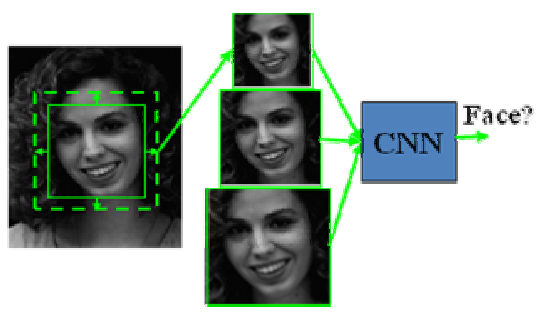

Fig. 3 - Face candidates' verification process using the proposed method.

According to the proposed face candidates' verification method the face candidate's image is extended and a pyramid of images across a scale is built for this extended area. This pyramid consists of the extended face candidate's images scaled by 
factor 0.8 . The size of the last pyramid's image has to be not less than $32 \times 36$ (default CNN's input size). Each image from the pyramid is processed by the $\mathrm{CNN}$ at once instead of been scanned by a fixed-size window like in the second known verification method. After the processing of all pyramids images by a CNN the number of multiple detections is counted and a candidate is accepted as a face when the number of multiple detections is equal or greater than the threshold value which is chosen experimentally. It's obviously that the number of CNN's simulations for one face candidate is

$$
\operatorname{NNSim}(x)=\sum_{s=0}^{S-1} W I N^{s} .
$$

The computational complexity's analysis of the proposed and known verification methods is showed in Table 1.

Table 1. Computational complexity comparison of the proposed and known verification methods used in the combined cascade of classifiers on CMU test set

\begin{tabular}{|l|c|c|}
\hline Verification method & $\begin{array}{c}\text { Total time of } \\
\text { CNN's } \\
\text { simulations, } \\
\text { s }\end{array}$ & $\begin{array}{c}\text { Fraction of } \\
\text { CNN's } \\
\text { simulations in } \\
\text { FD process, \% }\end{array}$ \\
\hline First & 0.7 & 3.1 \\
\hline $\begin{array}{l}\text { First (with multiple } \\
\text { detections' checking) }\end{array}$ & 5.0 & 18.0 \\
\hline Second & 81.6 & 79.3 \\
\hline Proposed & 9.4 & 30.2 \\
\hline
\end{tabular}

As we can see from Table 1, the first known verification method has the lowest computational complexity and increases the processing time of the combined cascade of classifiers by $3.1 \%$ (or $18 \%$ in the case of multiple detections' checking) on CMU test set. The largest computational complexity is inherent in the second known verification method which enlarges the processing time of the combined cascade of classifiers by $79.3 \%$. The proposed verification method increases the processing time by $30.2 \%$.

We also researched these verification methods according to the detection rate and number of FP (Fig. 4). The proposed verification method, as shown in Fig. 4, allows receiving in average up to $5.2 \%$ higher detection rate in comparison with the first known verification method with multiple detections' checking and only in average up to $0.2 \%$ lower detection rate as compared with the rate of the second known verification method. Taking into account the results of the abovementioned researches it is evidently that the proposed verification method is more suitable for the combined cascade of classifiers because it provides a high detection rate and allows boosting the FD process in 8.6 times in comparison with the second known verification method.

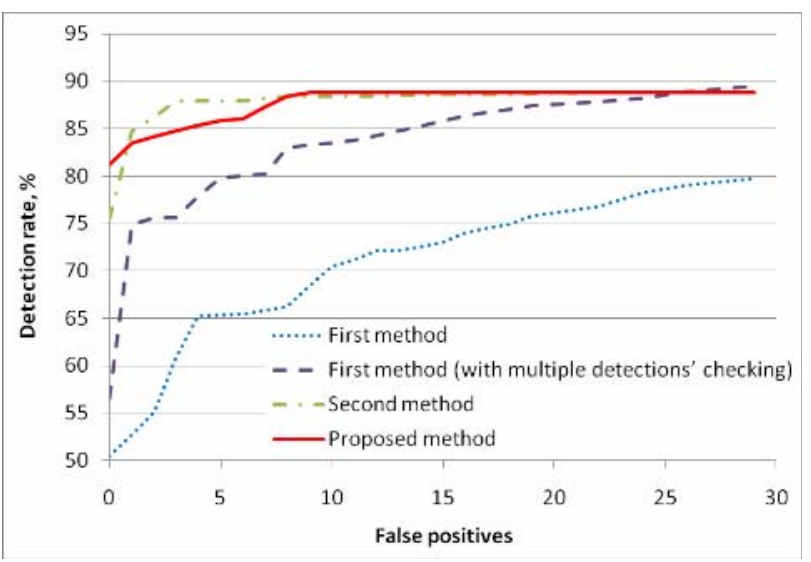

Fig. 4 - ROC-curves of the combined cascade of classifiers using different verification methods on CMU test set

The further experimental researches showed that the combined cascade of classifiers with the proposed face candidates' verification method demonstrates one of the best detection rates on CMU test set in comparison with known FD approaches (Fig. 5).

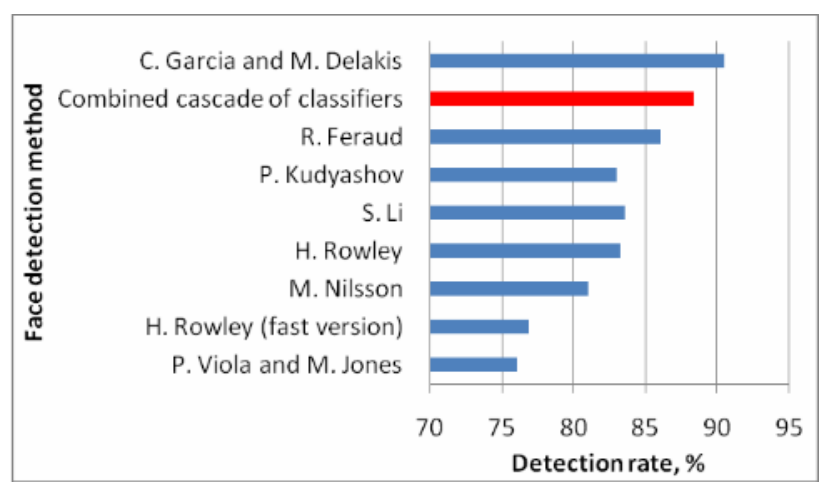

Fig. 5 - Comparison of the face detection methods' results using $\mathrm{CMU}$ test set at 7-10 false positives.

The improved FD method based on the combined cascade of classifiers yields only to the method of C. Garcia and M. Delakis [5] approximately on $2 \%$ by detection rate but it is 8 times faster.

\section{FACE DETECTION ON COLOR IMAGES}

The presence of information about skin color potentially can increase the efficiency of the FD process as it restricts the area of faces searching. As the result, it will reduce the number of false positive detections as well as the processing time of the input image $[22,23]$. Thus, there is a reason to improve a method based on combined cascade of classifiers for face detection in color images. It is necessary to add an additional level of face candidates' detection to the combined cascade of classifiers before the skin color based cascade of weak classifiers [17]. Taking 
into account that skin color segmentation can be pixel-based or region-based, the authors make use a pixel-based segmentation. It supposes to create a classifier which will distinguish the skin color pixels from the background ones. Also it is reasonable to make use of modeling method with the explicitly defined skin regions as it is simple in implementation, fast and quite accurate [22].

There are several color spaces that can be successfully used for segmentation. For example, for RGB color space the authors use the following explicitly defined boundaries of the skin color cluster (for each of the R, G, and B channel) [24]:

$\%$ The skin color model at uniform daylight illumination

$$
\begin{gathered}
R>95 \text { and } G>40 \text { and } B>20 \text { and } \\
\max \{R, G, B\}-\min \{R, G, B\}>15 \text { and } \\
|R-G|>15 \\
\text { and } R>G \text { and } R>B \\
\text { OR }
\end{gathered}
$$

\% The skin color model under flashlight lateral illumination

$$
\begin{aligned}
& |R-G| \leq 15 \text { and } \\
& R>B \text { and } G>B .
\end{aligned}
$$$$
R>220 \text { and } G>210 \text { and } B>170 \text { and }
$$

Also authors researched the TSL, YCbCr and YIQ color spaces and defined the following skin color cluster boundaries (for each of the T; $\mathrm{Cb}, \mathrm{Cr}$; I and Q channels) [23]:

$$
\begin{gathered}
0.45 \leq T \leq 0.65, \\
85 \leq C_{B} \leq 135 \text { and } 135 \leq C_{R} \leq 160, \\
0.02 \leq I \leq 0.22 \text { and }-0.08 \leq Q \leq 0.12 .
\end{gathered}
$$

The experimental results of FD method on color images were performed on the UCD test set [25], which consists of 58 color images with 224 upright frontal faces. On the Fig. 6 there are shown the experimental results of combined cascade and skin color segmentation in RGB, TSL, YCbCr and YIQ color spaces.

As you can see from the Fig. 6a, the highest detection rate was showed by TSL-based skin color segmentation. In comparison with YCbCr, YIQ and RGB the results are better in average by $0.4 \%, 1.3 \%$ and $2.7 \%$ representatively. From the Fig. $6 \mathrm{~b}$ we can conclude that the shortest time of FD is for the RGB color space.

The authors also have researched the effect on the detection rate of the combined cascade of classifiers for skin-color segmentation in two color spaces. The segmentation results are combined by logical operator AND (Fig. 7).

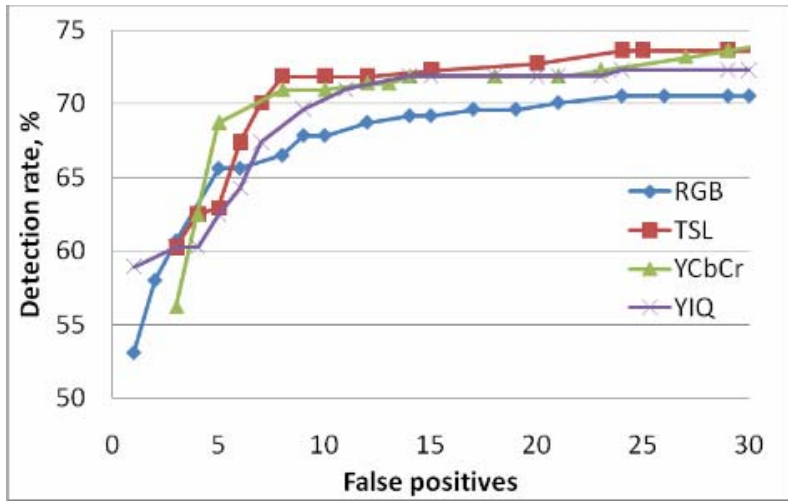

a)

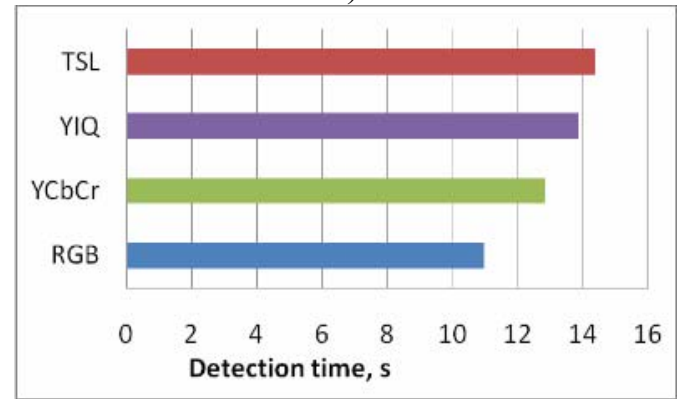

b)

Fig. 6 - ROC-curves (a) and the detection time (b) of the combined cascade of classifiers on UCD test set using different color spaces.

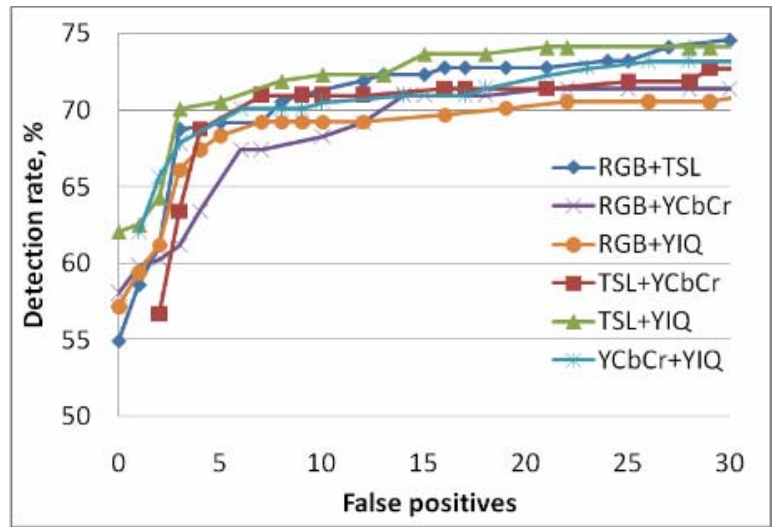

a)

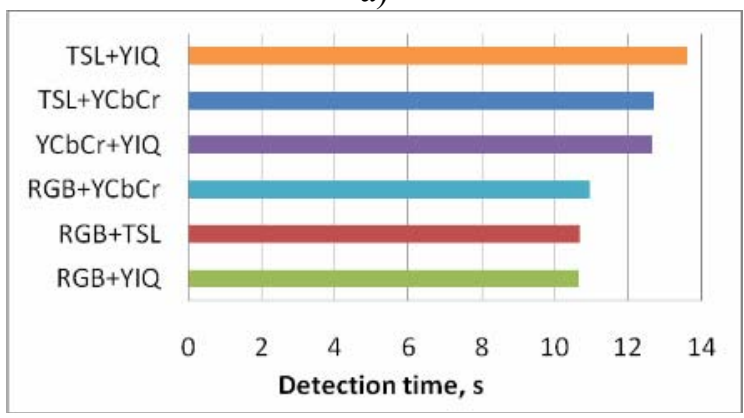

b)

Fig. 7 - ROC-curves (a) and the detection time (b) of the combined cascade of classifiers on UCD test set using combinations of color spaces.

From the FD results in Fig. 7a is it clear that to achieve high detection rate it's reasonable to use a combination of TSL+YIQ color spaces. Whereas to achieve the high speed (see Fig. 7b) we have to use a 
combination of RGB+TSL color spaces. It also should be noted that the RGB+TSL color spaces at the average show lower detection rate only by $1.3 \%$ in comparison with TSL+YIQ color spaces.

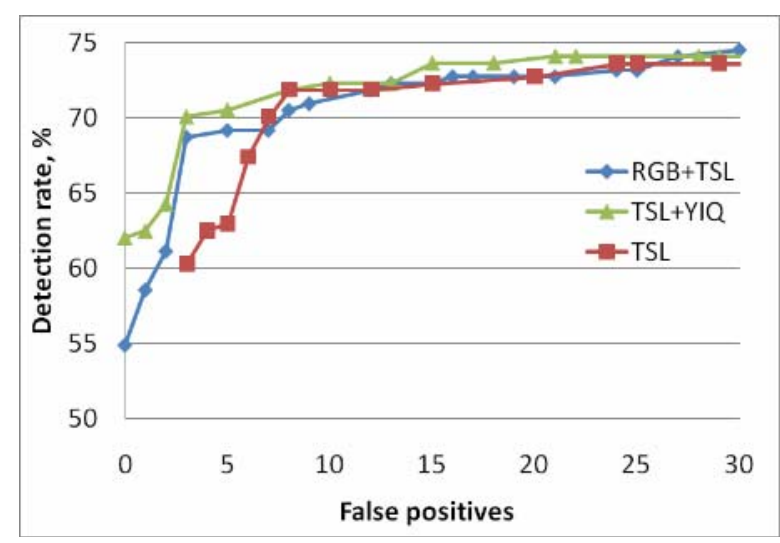

a)

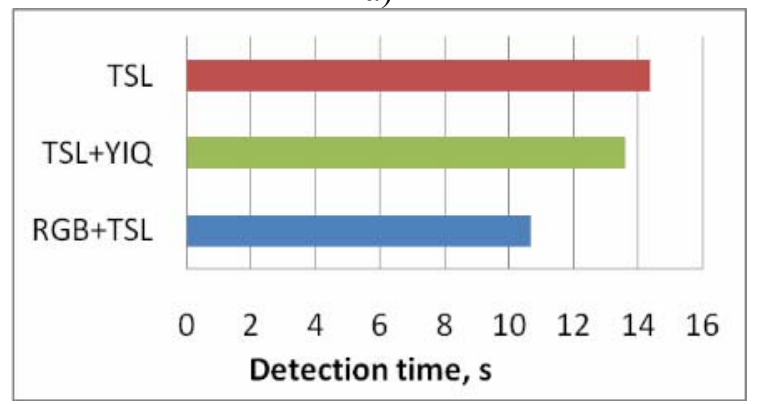

b)

Fig. 8 - ROC-curves (a) and the detection time (b) of the combined cascade of classifiers on UCD test set using one color space and their combinations.

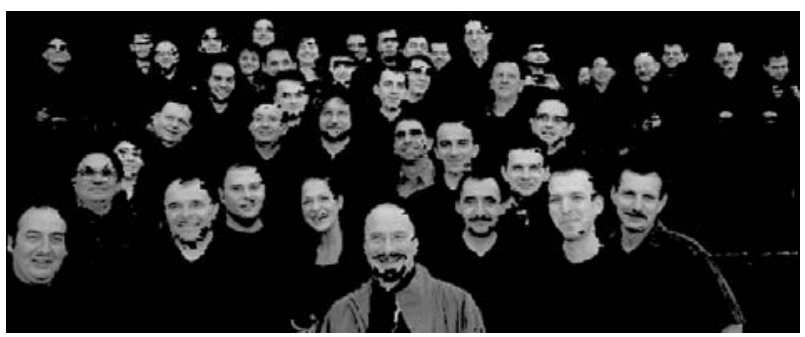

a)

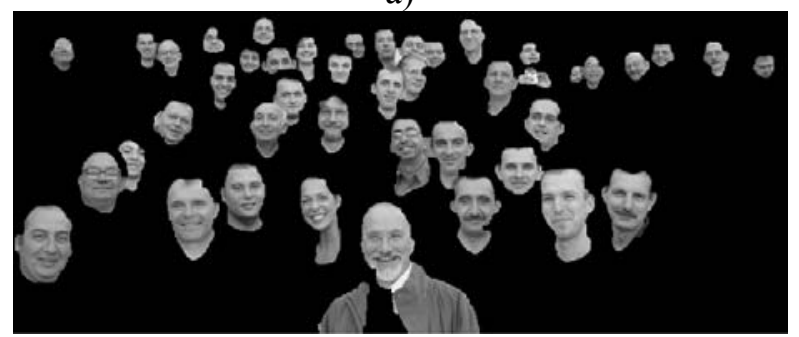

b)

Fig. 9 - The segmented image before (a) and after (b) applying of morphological operations

The analysis of FD results with the usage of skin color segmentation in one color space and in combination of few color spaces shows (Fig. 8) that it is more reasonable to use combination of TSL+YIQ or RGB+TSL color spaces. Such approach allows obtaining higher results of FD in average by $1.7 \%$ and $0.8 \%$ accordingly. Also it allows reducing the detection time on UCD test set by $6 \%$ and $26 \%$ respectively in comparison with single TSL color space.

In order to improve the segmentation results authors used the morphological operations such as erosion, dilation and holes' filling [17, 23]. The operation of erosion will cause replacing of the boundary pixels with 0 that allows distinguishing elements from each other. The dilation will perform the backward operation by replacing boundaries pixels of background with 1 . Holes' filling allows having solid segments without any background area inside. As a result of applying morphological operations the segments will be distinguished from each other, their boundaries will be rounded out and the small-size segments will be removed (Fig. 9).

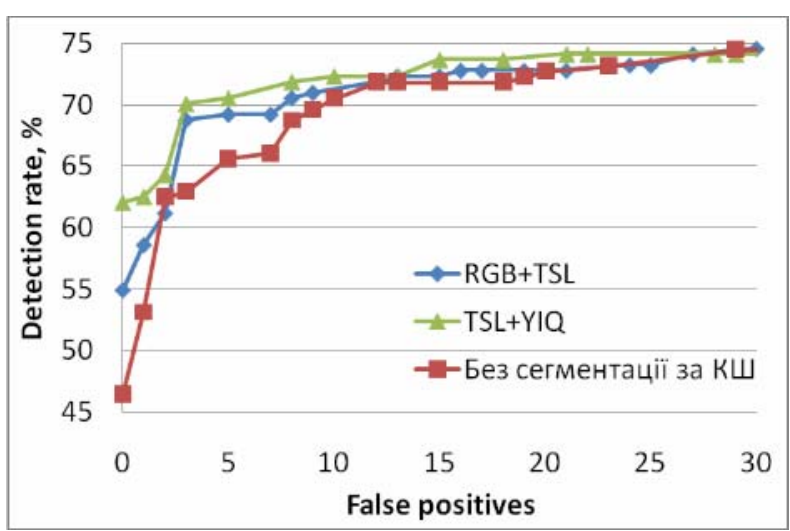

a)

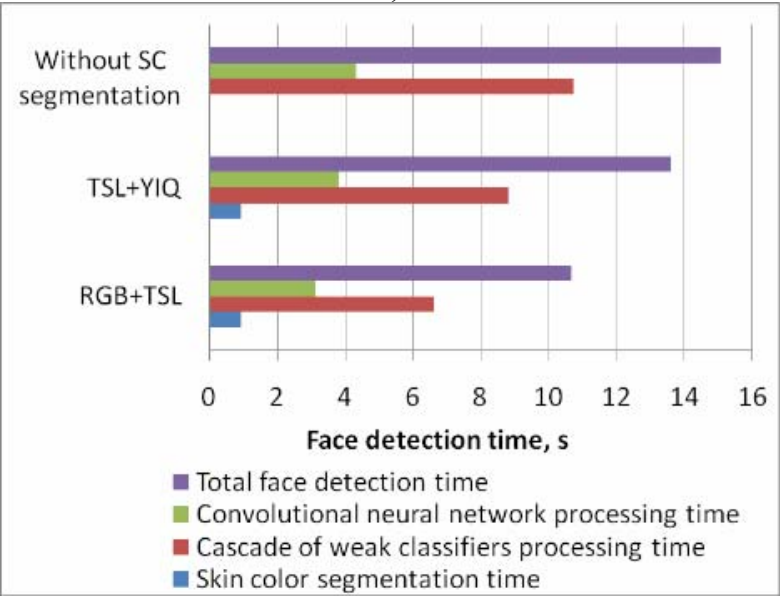

b)

Fig. 10 - ROC-curves (a) and the processing time (b) of the combined cascade of classifiers on UCD test set with and without skin color segmentation.

The further processing of the segmented image can be done either by passing each of the skin-like segments to the combined cascade of classifiers as the separate images or processing the entire image with the applied binary mask by combined cascade of classifiers. The mask consists of 1 where the pixel belongs to skin-color and 0 otherwise. The second approach is more preferable since it requires less 
computations - there are no need to find an integral image for each segment, the segments' area are not processing twice when the large segments include smaller ones. Also such approach allows detection of more face-candidates as the segments' boundaries can contain partially segmented faces and they will be processed too. In this case to avoid background processing the cascade of weak classifiers will process only those windows where the number of zero pixels is less than $70 \%$. Such high threshold level is explained by the necessity of processing skin-color segments' boundaries since the faces are often segmented partially. The experimental results of FD method on UCD test set show that processing of the input image by combined cascade of classifiers with the applies binary mask performs by $20 \%$ faster than the processing of each segments separately.

The authors also conducted a comparative analysis of FD results by the combined cascade of classifiers with and without skin-color segmentation (Fig. 10).

As we can see from Fig.10, the improved FD method with skin-color segmentation shows higher detection rate in overage by $1.3 \%$ and $2.6 \%$. Also it requires by $29 \%$ and $10 \%$ less time for input image processing when using RGB+TSL and TSL+YIQ color spaces respectively in comparison with FD method on grayscale images (see Section 2). Increasing of the detection rate caused by decreasing number of false positive detections since a lot of background areas where FP detections usually appear are rejected by skin-color segmentation level.

\section{SELECTION OF THE FACE DETECTION SYSTEM'S PARAMETERS}

The FD system was implemented in $\mathrm{C}++$ within Microsoft Visual Studio Team System 2008 environment [26] using Intel Open Computer Vision Library (OpenCV) [27] and Intel Integrated Performance Primitives (IPP) [28] (Fig. 11).

To determine the rational parameters with the maximal face detection rate, speed, and the minimal FP number authors performed a number of experiments on the test sets and in real environment, such as:

1) Determination of the number of active training epochs for CNN;

2) Comparative evaluation of the grayscale image preprocessing;

3) Determination of the multiple detections threshold values for the combined cascade of classifiers' levels at the grayscale and color image processing;

4) Test set analysis for the second kind error (the faces are not detecting);
5) Evaluation of the combined cascade of classifiers' performance while processing the video stream.

For testing there were used a Logitech QuickCam Messenger web camera, connected to the PC Intel Celeron E1200 Dual-Core 1.6GHz with 1Gb RAM.

1) In order to evaluate the required number of active training epochs for CNN for achieving acceptable detection rates and number of FP detections authors researched the dynamic of these parameters on the CMU test set during the training (Fig. 12).

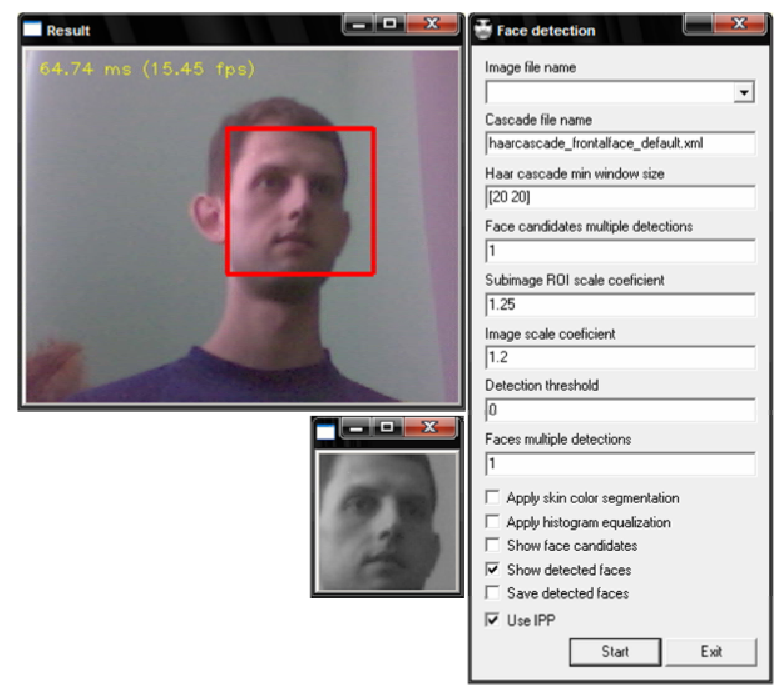

Fig. 11 - The main dialog box and the results of the developed face detection system

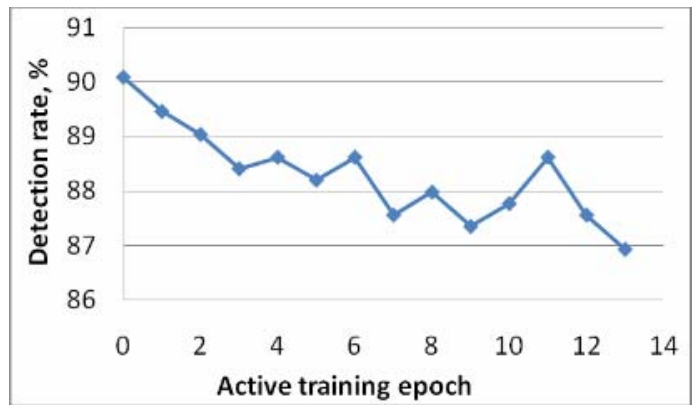

a)

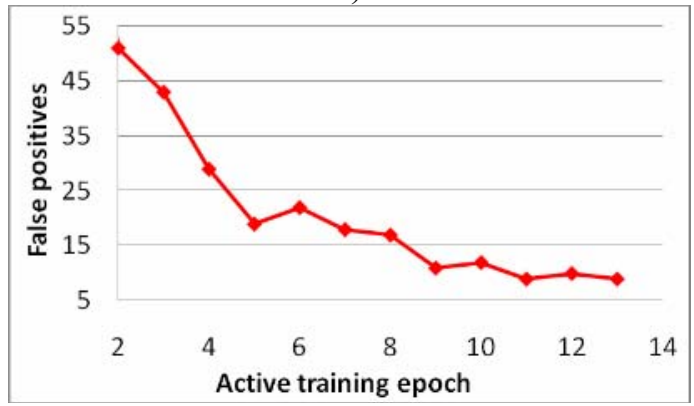

b)

Fig. 12 - Dependency of the detection rate (a) and FP number (b) from CNN active training epochs on CMU test set.

As follows from the training scenario on Fig. 12, 
the detection rate is gradually decreasing from $90 \%$ to $87 \%$ in 14 training epochs (by $0.2 \%$ per epoch in average). Thus, based only on the detection rate dynamic's analysis, the authors can conclude that to provide highest detection rate the training time should be minimal. However, the detection rate index should be considered always in combination with the number of FP detections. That's why to achieve an acceptable number of FP detections (1012) the CNN requires more than 8 training epochs.

2) Some FD methods $[2,3]$ expect to receive a preprocessed image at the input of classifier. Such preprocessing includes illumination correction and pixels' histogram equalization. As there was mentioned before, there are no need in preprocessing stage for improved FD method on grayscale images. The experiments showed that the pixels' histogram equalization of the face-candidates reduces the combined cascade of classifiers' detection rate on the CMU test set in average by $6 \%$ (Fig. 13).

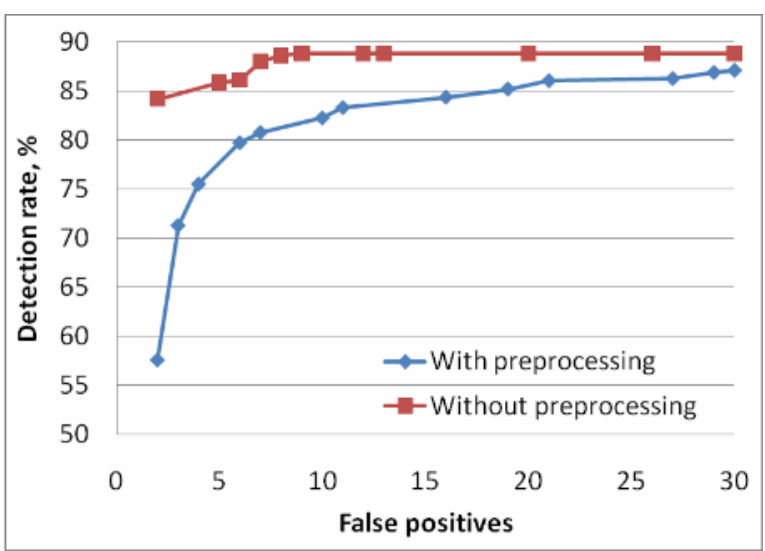

Fig. 13 - ROC-curves of the combined cascade of classifiers on CMU test set with and without input image preprocessing

3) While detecting faces no less important parameter is a threshold value of face-candidates multiple detections by cascade of weak classifiers and CNN. The threshold value affects on the correlation of detection rate and number of FP detections. It is obvious, that for the cascade of weak classifiers we should determine the value of this parameter that will provide highest detection rate. The reason is that the detection rate of cascade of weak classifiers determines the maximum possible detection rate of whole combined cascade of classifiers. The experiments showed (Fig. 14) that for cascade of weak classifiers it is reasonable to choose a threshold value of face-candidates multiple detections equal to 1 . Such value allows achieving of the highest detection rate up to $91 \%$.

The similar researches on determination of the multiple detections threshold value (MinMD) were also conducted for CNN (Fig. 15). As illustrated on Fig. 15, the maximum detection rate is achieved by setting the minimal number of multiple detections MinMD $=1$. In this case the detection rate is higher in comparison with other multiple detections values in average by $0.2-1.4 \%$. We should mention also that the usage of smaller MinMD $=1$ allows improving of the combined cascade of classifiers' performance due to applying the face acceptance system which is implemented in face-candidates verification module.

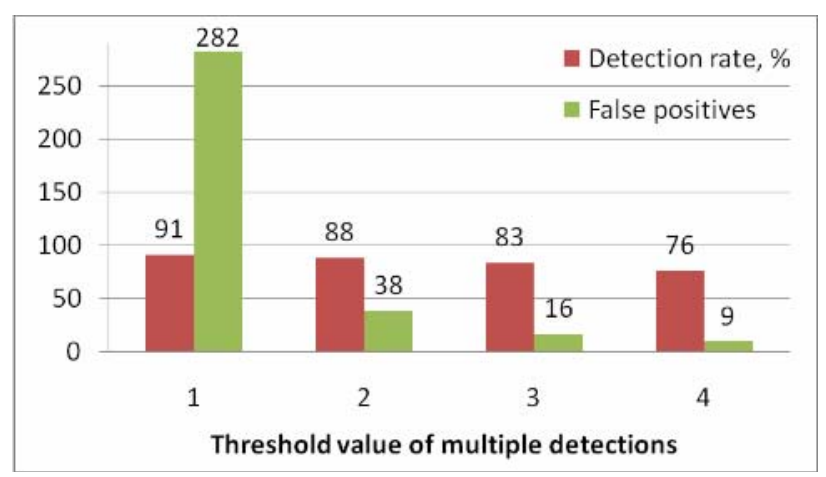

Fig. 14 - The detection rate of the cascade of weak classifiers on CMU test set with different multiple detections threshold values.

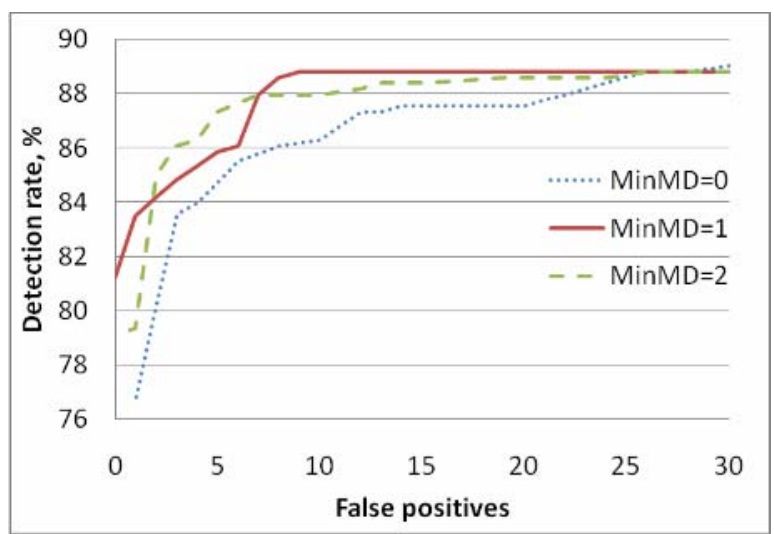

Fig. 15 - ROC-curves of the combined cascade of classifiers on CMU test set with different multiple detections threshold values for $\mathrm{CNN}$.

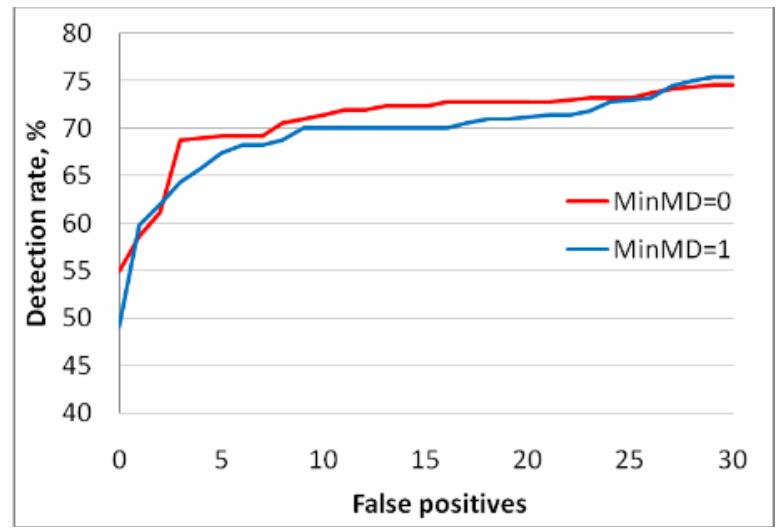

Fig. 16 - ROC-curves of the combined cascade of classifiers on UCD test set for different multiple detections threshold values for $\mathrm{CNN}$.

Taking into account that preliminary experiments on determination of the minimum number of multiple detections were performed on CMU test set 
with the grayscale images, it is expedient to determine the threshold value of this parameter on color images also for the cases where skin-color segmentation is performed. The experimental results were performed on UCD test set (Fig. 16).

It is more reasonable to set the threshold value to 0 when processing the color images by CNN. This allows to achieve higher detection rate on average by $1.3 \%$ than at MinMD=1 (see Fig. 16). Reducing the minimum number of multiple detections while processing the color images (in other words the stringency of cascade of combined classifiers) is explained by additional applying of skin color segmentation which rejects much of the background - potential FP detections.

4) While analyzing the error of the second kind it is necessary to keep in mind that the combined cascade of classifiers is specified for frontal horizontal faces' detection and the possible invariance to in- and out-of-plane rotations is determined by the nature of classifiers (cascade of weak classifiers or $\mathrm{CNN}$ ) and the train set. Particularly, the train set consist samples with rotations within $\left[-40^{0} ;+40^{0}\right]$ out of the plane, i.e. close to half profile, and the slopes in range $\left[-20^{\circ}\right.$; $\left.+20^{0}\right]$. Therefore, it is obviously that the combined cascade of classifiers should detect faces in these boundaries from the test set. Fig. 17 illustrates the examples of faces from CMU test set which were classified by mistake as non-faces.

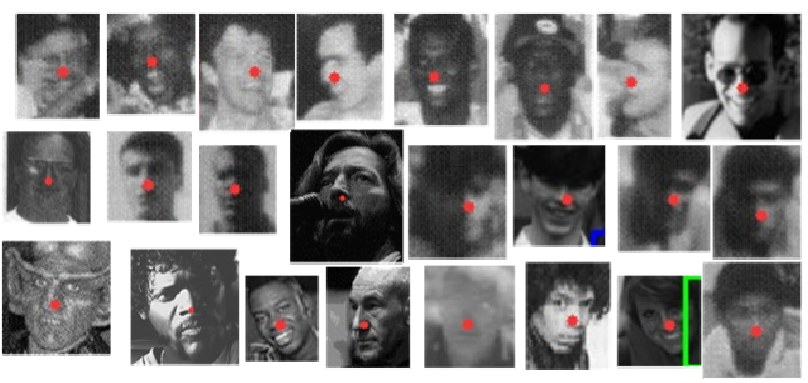

Fig. 17 - Some positive examples from CMU test set erroneously classified as non-faces by the combined cascade of classifiers.

Some erroneously classified faces have bigger inand out-of-plane rotation angles as it was provided in train set. Also some of them are blurred or obtained in difficult illumination conditions.

5) The combined cascade of classifiers' performance analysis of video flow processing showed 15-20 fps on the $352 \times 288$ pixels input image with 20x20 pixels minimal window size.

\section{CONCLUSIONS AND FUTURE RESEARCHES}

Human face detection method for grayscale images is improved using the combined cascade of classifiers which consists of Haar-like features' cascade of weak classifiers and convolutional neural network. The new face candidates' verification method for the combined cascade is proposed which uses the property of the convolutional neural network to handle an input image of any size at once. The improved face detection method based on the combined cascade of classifiers yields only to the method of C. Garcia and M. Delakis [5] approximately on $2 \%$ by detection rate but it is 8 times faster.

The improved face detection method is extended also to process color images by additional using of the mixture of two color spaces for skin color segmentation. The application of TSL+YIQ or RGB+TSL color spaces' combinations allows obtaining higher face detection results in average by $1.7 \%$ and $0.8 \%$ on UCD test set. Also it allows reducing the detection time by $6 \%$ and $26 \%$ respectively in comparison $\mathrm{w}$ single TSL color space.

The rational parameters of the face detection system are determined during the series of experiments in order to maximize a face detection rate and speed as well as to minimize the number of false positives.

Promising future research directions include the parallelization of the combined cascade of classifiers training using the computing cluster and application of the additional face candidates' detection means like motion segmentation.

\section{REFERENCES}

[1] Yang M. Recent Advances in Face Detection // IEEE ICPR 2004 Tutorial. - Cambridge, United Kingdom, 2004. - 93 p.

[2] Poggio T., Sung K. Example-based learning for view-based human face detection // IEEE Transactions on Pattern Analysis and Machine Intelligence. - 1998. - Vol. 20, No.1. - P. 3951.

[3] Rowley H., Baluja S., Kanade T. Neural network-based face detection // IEEE Transactions on Pattern Analysis and Machine Intelligence. - 1998. - Vol. 20. - P. 22-38.

[4] Yang M., Roth D., Ahuja N. A SNoW-Based Face Detector // Proceedings of Advances in Neural Information Processing Systems 12 (NIPS 12). - 2000. - P. 855-861.

[5] Garcia C., Delakis M. Convolution Face Finder: A Neural Architecture for Fast and Robust Face Detection // IEEE Transactions on Pattern Analysis and Machine Intelligence. 2004. - Vol. 26, Issue 11. - P. 1408-1423.

[6] Osuna E., Freund R., Girosi F. Training Support Vector Machines: An Application to Face Detection // Proc. of IEEE Conf. 
Computer Vision and Pattern Recognition. 1997. - P. 130-136.

[7] Romdhani S., Torr P., Schlkopf B., Blake A. Computationally efficient face detection // Proceedings of ICCV. - 2001. - vol. 1. - P. 695-700.

[8] Heisele B., Serre T., Prentice S., Poggio T. Hierarchical classification and feature reduction for fast face detection with support vector machines // Pattern Recognition. - 2003. 36(9). - P. 2007-2017.

[9] Schneiderman H., Kanade T. Probabilistic Modeling of Local Appearance and Spatial Relationships for Object Recognition // Proceedings of IEEE Conf. Computer Vision and Pattern Recognition. - 1998. - P. 45-51.

[10] Viola P., Jones M. Robust Real-Time Face Detection // International Journal of Computer Vision. 2004. - Vol. 57, No. 2. - P. 137-154.

[11] Li S., Zhang Z. FloatBoost Learning and Statistical Face Detection // IEEE Transactions on Pattern Analysis and Machine Intelligence. - 2004. - Vol. 26, No. 9. P. 1112-1123.

[12] Kudryashov P. Hybrid Human Face Detection Algorithm / P. Kudryashov, S. Fomenkov // Information Technologies. - 2007. - №10. - P. 20-23 (in Russian).

[13] Vetter T., Rätsch M., Romhani S. Efficient Face Detection by a Cascaded Support Vector Machine using Haar-like Features // Proceedings of The 26th German Association for Pattern Recognition Symposium (DAGM'04). - Tübingen (Germany), 2004. - P. 62-70.

[14] Zuo F., With P. Cascaded Face Detection Using Neural Network Ensembles // EURASIP Journal on Advances in Signal Processing. 2008. - Vol. 2008, Issue 1. - 13 p.

[15] Nilsson M., Nordberg J., Claesson I. Face Detection using Local SMQT Features and Split up Snow Classifier // Proceedings of the IEEE International Conference on Acoustics, Speech and Signal Processing (ICASSP 2007). - 2007. - Vol. 2. - P. 589-592.

[16] Paliy I. Human Face Detection Methods Using a Combined Cascade of Classifiers / I. Paliy // Computing. - 2008. - Vol. 7, Issue 1. - P. 114125 (in Ukrainian).

[17] Paliy I. Face Detection Method and Mean for Color Images Effective Processing / I. Paliy // Artificial Intelligence. - 2008. - Vol. 4. - P. 402-411 (in Ukrainian).

[18] Freund Y., Schapire R. A Decision-Theoretic Generalization of On-Line Learning and an Application to Boosting // Computational Learning Theory. - Springer-Verlag. - 1995. P. 23-37.
[19] LeCun Y., Bottou L., Bengio Y. GradientBased Learning Applied to Document Recognition // Intelligent Signal Processing, IEEE Press. - 2001. - P. 306-351.

[20] Wasserman A. Neural Computing: Theory and Practice. - New York: Van Nostrand Reinhold. $-1989 .-230 \mathrm{p}$.

[21] Simard P., Steinkraus D., Platt J. Best Practices for Convolutional Neural Networks Applied to Visual Document Analysis // Seventh International Conference on Document Analysis and Recognition (ICDAR'03). - Vol. 2. - 2003. - P. 958.

[22] Vezhnevets V., Sazonov V., Andreeva A. A Survey on Pixel-Based Skin Color Detection Techniques // Proceedings of Graphicon-2003. - Moscow (Russia), 2003. - P. 85-92.

[23] Paliy I. Improved Neural Network-based Face Detection Method using Color Images / I. Paliy, Y. Kurylyak, A. Sachenko, K. Madani, A. Chohra // Proceedings of the Third International Workshop on Artificial Neural Networks and Intelligent Information Processing (ANNIIP 2007). - Angers (France), 2007. - P. 107-114.

[24] Peer P., Kovac J., Solina F. Human Skin Colour Clustering for Face Detection // EUROCON 2003 - International Conference on Computer as a Tool. - Ljubljana (Slovenia), 2003. - Vol. 2. - P. 144-148.

[25] Sharma P., Reilly R. A Color Face Image Database for Benchmarking of Automatic Facial Detection Algorithms // Proceedings of 4th European Conference of Video/Image Processing and Multimedia Communications. 2003. - P. 423-428.

[26] Microsoft Visual Studio Team System 2008 product page: http://msdn.microsoft.com/enus/vsts2008/products/default.aspx.

[27] OpenCV library: http://sourceforge.net/ projects/opencv/.

[28] Intel IPP library: http://www.intel.com/cd/software/products/asm o-na/eng/302910.htm.

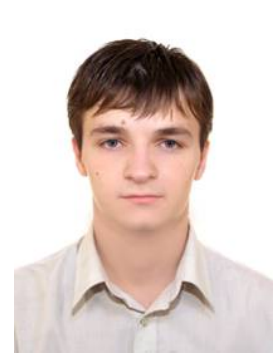

Yuriy Kurylyak received his Bachelor Degree in computer engineering (2005) and his Master Degree in computer systems and networks (2006) from Ternopil National Economic University (TNEU) Ternopil, Ukraine.

From January till November 2006 he worked as an engineer at the ComputerAided Design Laboratory, Faculty of Computer Information Technologies, TNEU. Since November 
2006 he is a Ph.D. Student. From May 2007 he is a junior scientist at the Research Institute of Intelligent Computer System (ICS), TNEU. He is a member of Intelligent Robotic Systems Research Group at ICS.

His main research interests are Image and Video Processing, Motion Detection, Artificial Intelligence, Neural Networks.

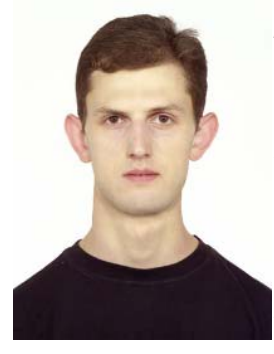

Ihor Paliy. Scientific associate of Research Institute of Intelligent Computer Systems, Ternopil National Economic University (TNEU), Ternopil, Ukraine.

Received the specialist's diploma of Information Systems in Management in 2002, master diploma in Economic Cybernetics in 2003from TNEU. From 2004 till now he is a PhD. student of the Department of Information Computing Systems and Control, TNEU.

His research interests are computer vision, pattern recognition, image processing, machine learning.

\section{Anatoly Sachenko is Professor} and Head of the Department of Information Computing Systems and Control and Director of American-Ukrainian Program in Computer Science, Ternopil State Economic University. He earned his B.Eng. Degree in Electrical Engineering at L'viv Polytechnic Institute in1968 and his PhD Degree in Electrical Engineering at L'viv Physics and Mechanics Institute in 1978 and his Doctor of Technical Sciences Degree in Electrical and Computer Engineering at Leningrad Electrotechnic Institute in 1988. Since 1991 he has been Honored Inventor of Ukraine, since 1993 he has been IEEE Senior Member.

His main Areas of Research Interest are Implementation of Artificial Neural Network, Distributed System and Network, Parallel Computing, Intelligent Controllers for Automated and Robotics Systems. He has published over 300 papers in areas above.

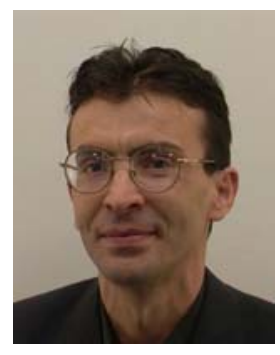

Dr. Amine Chohra. Received his Doctorate es-sciences in 1999 from Ecole Nationale Polytechnique, Algiers (Algeria). He has been a member of Artificial Intelligence and Robotics Laboratory (LRIA) of CDTA from 1991 to 1999. From 1999 to 2001, he worked as post doctoral researcher, with Behavior Engineering team of AiSGMD, Sankt Augustin (Germany) and with Dependable Computing Group of IEI-CNR, Pisa (Italy), respectively. From 2001 2003, he has been teacher/researcher at Orleans University, ENSI de Bourges (France), and a member of Vision and
Robotics Laboratory (LVR / UPRES EA 2078).

Since September 2003, he is Assistant Professor at Senart Institute of Technology of PARIS XII University, Lieusaint (France) and a staff member of Image, Signal, and Intelligent Systems Laboratory (LISSI / EA 3956) of this University.

His research interests are information processing systems, knowledge based systems, hybrid intelligent systems, soft computing, machine learning and decision-making, pattern recognition and computer aided diagnosis.

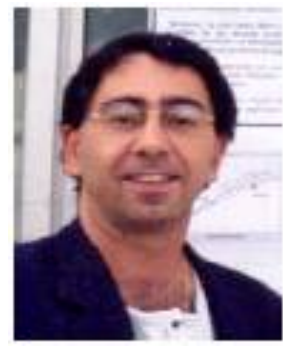

Prof. Kurosh Madani. Received his Ph.D. degree in Electrical Engineering and Computer Sciences from University PARIS XI, Orsay, France, in 1990. From 1989 to 1990, he worked as assistant professor at Institute of Fundamental Electronics of PARIS XI University. In 1990, he joined CreteilSenart Institute of Technology of University PARIS XII - Val de Marne, Lieusaint, France, where he worked from 1990 to 1998 as assistant professor. In 1995, he received the DHDR Doctor Habilitate degree (senior research Dr. Hab. degree) from University PARIS XII - Val de Marne. Since 1998 he works as Chair Professor in Electrical Engineering of Senart Institute of Technology of University PARIS XII.

From 1992 to 2004 he has been head of Intelligence in Instrumentation and Systems Laboratory (I2S / JE 2353) located at Senart Institute of Technology. Since 2005, he is head of one of the three research groups of Image, Signal and Intelligent Systems Laboratory (LISSI / EA 3956) of PARIS XII University. He has worked on both digital and analog implementation of processors arrays for image processing, electro-optical random number generation, and both analog and digital ANN implementation.

His current research interests include large ANN structures modeling and implementation, hybrid neural based information processing systems and their software and hardware implementations, design and implementation of real-time neurocontrol and neural based fault detection and diagnosis systems. Since 1996 he is a permanent member (elected Academician) of International Informatization Academy. In 1997, he was also elected as Academician of International Academy of Technological Cybernetics. 